\title{
A MULTI-SCALE METHOD FOR BIOLUMINESCENCE TOMOGRAPHY USING MULTIPLE TYPES OF A PRIORI INFORMATION
}

\author{
Yujie Lv, Jie Tian*, Wei Yang, Chenghu Qin \\ Institute of Automation \\ Chinese Academy of Sciences \\ P. O. Box 2728, Beijing 100080, China
}

\author{
Wenxiang Cong, Ge Wang ${ }^{\dagger}$ \\ School of Biomedical Engineering \& Sciences \\ Virginia Polytechnic Institute \& State University \\ Blacksburg, VA 24061, USA
}

\begin{abstract}
Bioluminescence tomography (BLT) is a novel in vivo technique which may reveal the molecular and cellular activity at the whole-body level in small animal studies. In order to reconstruct bioluminescence sources accurately, it is necessary to incorporate all types of a priori information available and develop novel reconstruction algorithms. In this paper, a multi-scale method is proposed for BLT based on adaptive finite element analysis. Specifically, multiple constraints, including multi-spectral measurements, anatomical and optical knowledge, are imposed to deal with the solution nonuniqueness of BLT. Also, adaptive finite element meshing and permissible source region refinement are utilized to help improve the reconstruction quality and speed. Finally, with the micro-MRI based virtual mouse phantom (MOBY) and the Monte Carlo based synthetic data, the feasibility and effectiveness of this BLT approach is demonstrated.
\end{abstract}

Index Terms - Bioluminescence tomography (BLT), Finite element methods, adaptive meshing, knowledge-based reconstructioin

\section{INTRODUCTION}

Although bioluminescence imaging, as a small animal imaging modality, has been rapidly developed and applied in the past several years [1], tomographically observing the molecular and cellular activities at the whole-body small animal level, that is, bioluminescence tomography (BLT) [2], still faces challenging problems to be solved. Theoretically, three dimensional bioluminescent source reconstruction is an inverse source problem, which has been less researched and is different from the inverse scattering imaging, such as diffusion optical tomography (DOT). In the highly heterogeneous biological tissues, the scattering and absorption of the photon emitted by bioluminescent source further increases the difficulty of source localization. In addition, though the absence

\footnotetext{
${ }^{*}$ Work supported by NBRPC(2006CB705700), NSFDYS (60225008) and NSFC (30370418, 30500131 and 60532050) in China

${ }^{\dagger}$ Work supported by an NIH/NIBIB grant EB001685
}

of external illumination sources acquires a high-sensitive signal, it complicates the tomographic problem. Therefore, the unique and accurate reconstruction of bioluminescent source and the development of a fast and robust tomographic algorithm are topics for further investigation [3].

Based on diffusion approximation theory, the uniqueness theorem shows that the BLT solution is not unique in the general case [4]. It is necessary to incorporate a priori information for accurate BLT reconstruction. Taking into account the surface light power distribution and the heterogeneous structure of the phantom, the BLT reconstruction methods employing a priori permissible source region are developed[5, 6]. Then, in view of the spectral characteristics of the underlying bioluminescent source, hyper- and multi-spectral BLT methods are also proposed [7,8]. Note that the assumption of a homogeneous optical background is inadequate for the spectrally resolved bioluminescence tomography, especially for deeper bioluminescent source in the small animal [8].

In this paper, a novel multi-scale tomographic algorithm employing adaptive finite element is proposed and developed for BLT reconstruction. This method considers anatomical structure and bioluminescent spectrum as a priori information to constrain the possible solution of BLT. Adaptive mesh refinement and permissible source region evolution based on a posteriori measures not only reduces the ill-posedness of the BLT problem remarkably, but also avoids the dimensional disaster arising from the utilization of multi-spectral data. In the numerical reconstruction, a micro-MRI based real mouse volume is employed to provide the anatomical information [9] and the optical properties corresponding to the segmented tissues are also determined through a priori experimental results [8]. In order to acquire the measured data, the multi-spectral measurements are also generated through a modified molecular optical simulation environment (MOSE) that we developed based on Monte Carlo simulation. Reconstructed results show the merits and potentials of the proposed tomographic algorithm for practical BLT reconstruction.

The organization of the paper is as follows. The next section introduces the proposed BLT algorithm incorporating multiple a priori information and adaptive finite element. 
BLT reconstruction is thoroughly demonstrated in Section 3. Section 4 discusses the relevant issues and concludes the paper.

\section{ALGORITHMS}

\subsection{Formulation}

The propagation of bioluminescent photons in the biological tissue can be well modeled by the steady-state diffusion equation and Robin boundary condition $[5,7]$. Taking into account the influence of light wavelength $\lambda$ on tissue optical property, the following physical model is given:

$-\nabla \cdot(D(\mathbf{x}, \lambda) \nabla \Phi(\mathbf{x}, \lambda))+\mu_{a}(\mathbf{x}, \lambda) \Phi(\mathbf{x}, \lambda)=S(\mathbf{x}, \lambda)(\mathbf{x} \in \Omega)$

$\Phi(\mathbf{x}, \lambda)+2 A\left(\mathbf{x} ; n, n^{\prime}\right) D(\mathbf{x}, \lambda)(\mathbf{v}(\mathbf{x}) \cdot \nabla \Phi(\mathbf{x}, \lambda))=0(\mathbf{x} \in \partial \Omega)$

where $\Omega$ and $\partial \Omega$ are the domain and its boundary respectively; $\Phi(\mathbf{x}, \lambda)$ denotes the photon flux density; $S(\mathbf{x}, \lambda)$ is the source energy density; $\mu_{a}(\mathbf{x}, \lambda)$ is the absorption coefficient; $D(\mathbf{x}, \lambda)=1 /\left(3\left(\mu_{a}(\mathbf{x}, \lambda)+(1-g) \mu_{s}(\mathbf{x}, \lambda)\right)\right)$ is the optical diffusion coefficient, $\mu_{s}(\mathbf{x}, \lambda)$ the scattering coefficient, and $g$ the anisotropy parameter; $\mathbf{v}$ is the unit outer normal on $\partial \Omega$. In the practical experiment, if the outgoing flux density is detected with the filter of bandpass, the continuous spectral range of bioluminescent reporters is divided into several bands $w b_{k} \in\left[\lambda_{k}, \lambda_{k+1}\right], k=1,2, \ldots, K-1$.

In the framework of adaptive finite element analysis, the sequence $\left\{\mathcal{T}_{1}, \ldots \mathcal{T}_{l}, \ldots\right\}$ of nested triangulation of the given domain $\Omega$ and the corresponding linear finite element spaces $\left\{\mathcal{V}_{1}, \ldots \mathcal{V}_{l}, \ldots\right\}$ are defined. There are $N_{\mathcal{T}_{l}}$ elements and $N_{\mathcal{P}_{l}}$ vertex nodes at the $l$-th discretized level. We assemble over all the elements by the finite element method, the matrix form of Eqs. (1-1) and (1-2) is as follows:

$$
\left(K_{l}\left(w b_{k}\right)+C_{l}\left(w b_{k}\right)+B_{l}\left(w b_{k}\right)\right) \Phi_{l}\left(w b_{k}\right)=F_{l}\left(w b_{k}\right) S_{l}\left(w b_{k}\right)
$$

Let $M_{l}\left(w b_{k}\right)=K_{l}\left(w b_{k}\right)+C_{l}\left(w b_{k}\right)+B_{l}\left(w b_{k}\right)$, where $M_{l}\left(w b_{k}\right)$ is a symmetric positive-definite matrix. Considering the linear relationship between the unknown source variable $S_{l}\left(w b_{k}\right)$ and the boundary measured photon flux density $\Phi_{l}^{\text {meas }}\left(w b_{k}\right)$, we may obtain:

$$
\Phi_{l}^{\text {meas }}\left(w b_{k}\right)=\mathcal{F}_{l}\left(w b_{k}\right) S_{l}\left(w b_{k}\right)
$$

where $\mathcal{F}_{l}\left(w b_{k}\right)$ can be established by removing those rows of $\left[M_{l}\left(w b_{k}\right)^{-1} F_{l}\left(w b_{k}\right)\right]$ corresponding to the non-measurable points. Furthermore, the energy percentage of each spectral band $w b_{k}$ can be determined by the beforehand experimental spectral analysis of bioluminescent source, that is $S\left(w b_{k}\right)=$ $\omega\left(w b_{k}\right) S$, where $\omega\left(w b_{k}\right) \geq 0$ and $\sum_{k=1}^{K} \omega\left(w b_{k}\right) \approx 1, S$ denotes the total photon density. Taking into account the above spectral distribution and the selection of permissible source region, we have

$$
\Phi_{l}^{\text {meas }}=\mathcal{F}_{l} \mathcal{W}_{l}^{S} S_{l}
$$

where

$$
\Phi_{l}^{\text {meas }}=\left[\begin{array}{c}
\Phi_{l}^{\text {meas }}\left(w b_{1}\right) \\
\Phi_{l}^{\text {meas }}\left(w b_{2}\right) \\
\vdots \\
\Phi_{l}^{\text {meas }}\left(w b_{K}\right)
\end{array}\right], \mathcal{F}_{l}=\left[\begin{array}{c}
\omega\left(w b_{1}\right) \mathcal{F}_{l}\left(w b_{1}\right) \\
\omega\left(w b_{2}\right) \mathcal{F}_{l}\left(w b_{2}\right) \\
\vdots \\
\omega\left(w b_{K}\right) \mathcal{F}_{l}\left(w b_{K}\right)
\end{array}\right]
$$

$\mathcal{W}_{l}^{S}$ is the diagonal matrix and is used to select the permissible source region, that is:

$$
\begin{gathered}
\mathcal{W}_{l}^{S}=\operatorname{Diag}\left(w_{l(11)}^{s}, w_{l(22)}^{s}, \ldots, w_{l(i i)}^{s}, \ldots, w_{l\left(N_{P_{l}} N_{P_{l}}\right)}^{s}\right), \\
w_{l(i i)}^{s}= \begin{cases}0 & \left\{s_{l(i)}^{\prime}<\gamma_{s p}^{(l)} s_{l}^{\prime} \max , l>1\right\} \\
1 & \{l=1\} \text { or }\left\{s_{l(i)}^{\prime} \geq \gamma_{s p}^{(l)} s_{l}^{\prime} \max , l>1\right\}\end{cases}
\end{gathered}
$$

$s_{l(i)}^{\prime}$ and $s_{l}^{\prime \max }$ are the reconstructed results prolonged from the $(l-1)$ th level and the corresponding maximum, that is

$$
S_{l}^{\prime}=I_{l-1}^{l} S_{l-1}^{r}(l \geq 2)
$$

$I_{l-1}^{l}$ is the prolongation operator; $\gamma_{s p}^{(l)}$ is the ratio factor; and $S_{l-1}^{r}$ are the reconstructed results on the $(l-1)$ th level. By retaining those columns of $\mathcal{F}_{l} \mathcal{W}_{l}^{S}$ corresponding to the permissible source region $S_{l}^{p}$, the following linear system is obtained:

$$
\mathcal{A}_{l} S_{l}^{p}=\Phi_{l}^{\text {meas }}
$$

In BLT, the source density constraint may be utilized as a priori information. Therefore, the following $l$-th minimization $\Theta_{l}\left(S_{l}^{p}\right)$ is defined with Tikhonov regularization methods:

$$
\min _{0 \leq S_{l}^{p} \leq S_{l}^{\text {sup }}} \Theta_{l}\left(S_{l}^{p}\right)=\left\{\left\|\mathcal{A}_{l} S_{l}^{p}-\Phi_{l}^{\text {meas }}\right\|_{\Lambda}+\lambda_{l} \eta_{l}\left(S_{l}^{p}\right)\right\}
$$

where $S_{l}^{s u p}$ is the $l$-th level upper bound of the source density; $\Lambda$ is the weight matrix, $\|V\|_{\Lambda}=V^{T} \Lambda V ; \lambda_{l}$ the regularization parameter; and $\eta_{l}(\cdot)$ the penalty function. Here, we choose the modified spectral projected gradient based large-scale optimization algorithm to solve the above problem [10].

\subsection{Relevant issues}

Two different error estimations, that is, the direct maximum selection method and the hierarchical defect correction technique [6], are employed in the permissible and forbidden source regions. On a coarse mesh which brings few discretized elements in the permissible source region, the elements with higher values of the optimization results most likely represent actual source locations despite that they may look larger and darker than the actual. By selecting these elements for refinement, the BLT solution will be improved in general. Despite that the discrepancy between the measured data of different spectral bands is utilized to improve BLT reconstruction, the computational error distributions on discretized elements are consistent with each other due to the utilization of the same diffusion approximation and discretized domain. In 
order to reduce the computational burden, the error estimation on one single spectral band is performed in the forbidden source region. Therefore, the approximated error indicator $\tilde{e}_{\mathcal{V}_{l}}^{\mathcal{W}_{l}}\left(w b_{k}\right)$ is used to guide local mesh refinement, where $\mathcal{W}_{l}$ is the quadratic finite element space corresponding to $\mathcal{V}_{l}$. The detailed information can be found in Ref. [6].

Adaptive mesh refinement depends on the selection of domain triangulation. Although a significant collection of literature focuses on unstructured quadrilateral and hexahedral methods, automatic unstructured mesh generation algorithms, especially mesh acquisition from medical images in biomedical field, have lent themselves more readily to triangular and tetrahedral meshing. Here, we choose the tetrahedron as the basis element of the mesh and the red-green refinement strategy for refining the selected elements [11].

Although different objective functions need to be treated due to the adaptive mesh evolution and the selection of $a$ posteriori permissible source region, a final reasonable solution needs to be found, which makes the gradient norm $\left\|g_{\Theta_{l}}\left(S_{l}^{p}\right)\right\|$ approach 0 , where $g_{\Theta_{l}}\left(S_{l}^{p}\right)=\nabla \Theta_{l}\left(S_{l}^{p}\right)$. Considering the utilization of the selected optimization method, we select the norm ratio of the current gradient to the initial one and the iteration number on each level as the switch indexes. As far as the stopping criterion is concerned, we utilize the current gradient norm on the $l$-th level, the maximum number of mesh refinement or the discrepancy between the measured and computational boundary nodal flux data to evaluate if the whole reconstruction procedure should be terminated.

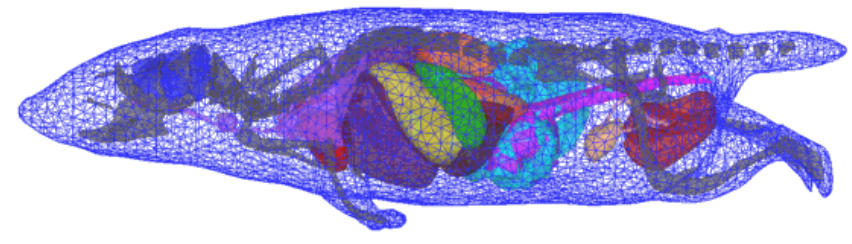

Fig. 1. The mesh view of the MOBY mouse phantom.

\section{RESULTS}

In this experiment, we assumed that the observed PC3M-Luc cells were tagged with firefly luciferase, and the cells emitted bioluminescent photons in the spectral range $[500 \mathrm{~nm}, 750 \mathrm{~nm}]$ [12]. In view of current measurement technology, we could divide the whole emission spectrum into five regions with discrete steps of $50 \mathrm{~nm}$.

As far as the mouse phantom was concerned, the first time gate of MOBY's image volume was used for BLT reconstruction [9]. The M3C algorithm with modified surface mesh smooth and simplification methods, and advancing front technique generates different scale meshes of the whole-body mouse phantom [13]. One of them is shown in fig. 1. In view of the non-scattering region of the mouse brain, only the mouse torso, from the neck to the base of the tail, was utilized. The organs defined by MOBY in the mouse torso were further dealt with due to the information absence of their optical properties from current literature data, which was the same with Ref. [8]. Then, the mouse phantom above utilized triangle elements to represent different organs, which included about 14600 triangle facets and approached the MOBY-defined organ shapes preferably. The modified MOSE platform utilized the surface mesh of the mouse phantom to generate the multi-spectral synthetic data. The initial coarse volumetric finite element mesh included 725 nodes and 3548 tetrahedral elements. The initial measured points were distributed on the mouse phantom's side surface, on which the multi-spectral measured data were calculated by the interpolation between the generated point values using MOSE.

When the bioluminescent source with $1.0 \mathrm{~mm}$ radius and 1.0 nano/Watts total power was located at half-radius from the mouse surface, figure 2 shows the reconstructed results based on the multi-scale tomographic algorithm. The whole reconstruction procedure took about 240 seconds on a PC with Intel processor (Pentium $42.8 \mathrm{GHz}$ ) and 1GB of RAM. Two adaptive mesh refinements were performed in the whole reconstruction procedure, and figure 3 shows the evolution of surface mesh and reconstructed results. When the reconstruction procedure started from the coarse mesh, the goal-oriented mesh refinement and the selection of a posteriori permissible source region not only brought the remarkable reduction of time cost, but also obtained desirable results.

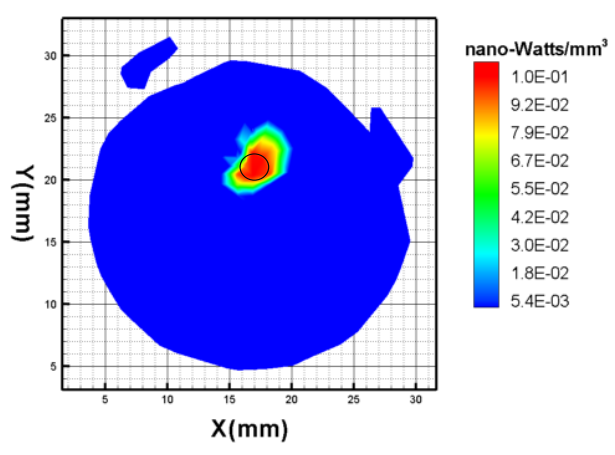

Fig. 2. BLT reconstruction when light source is placed at halfradius position of the mouse phantom. The black circle represents the actual source position.

\section{DISCUSSIONS AND CONCLUSION}

In recent years, compared with other molecular imaging modalities, bioluminescence imaging has been playing a pioneer role in preclinical studies due to the extensive application of bioluminescence in biology. As the transition from the verification experiment of simple phantoms to the biological re- 


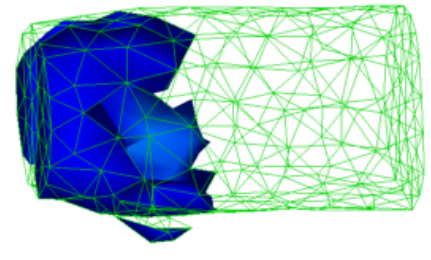

(a)

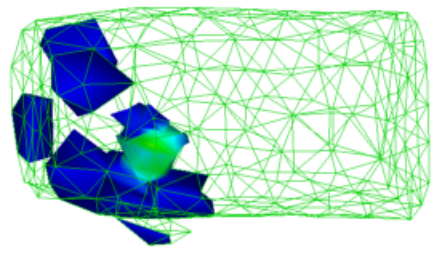

(b)

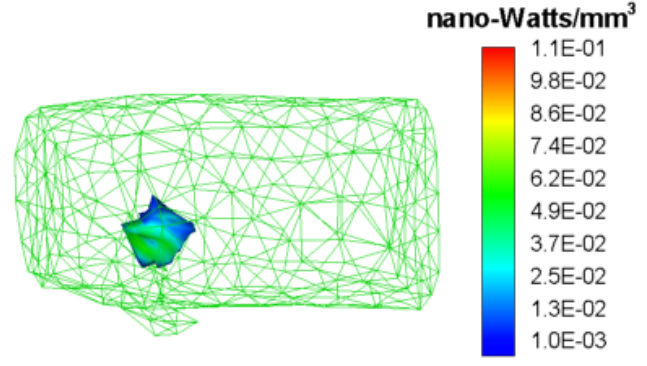

(c)

Fig. 3. The evolution of surface mesh and reconstructed results with bioluminescent source placed at half-radius position. Green mesh denotes the surface mesh of mouse phantom.

search using in vivo small animal is performed, rapid increase of measured data set and disposal of actual small animal models have brought a big challenge to the reconstruction speed, quality, robustness and stability of the tomographic algorithm. The multi-scale reconstruction provides a graceful strategy for improving the BLT reconstruction. Adaptive finite element algorithm not only has the favorable coarse grid correction feature, but also improves the reconstruction time and memory cost due to adaptive mesh evolution and reconstruction strategy based on the from-coarse-to-fine mesh sequence.

In conclusion, we have proposed and developed a novel multiscale tomographic algorithm to reconstruct the bioluminescent source distribution. The proposed tomographic algorithm provides a significant imaging method and leads to high-performance BLT investigations.

\section{REFERENCES}

[1] C. H. Contag and M. H. Bachmann, "Advances in bioluminescence imaging of gene expression," Annu. Rev. Biomed. Eng., vol. 4, pp. 235-260, 2002.

[2] G. Wang, E. A. Hoffman, G. McLennan, L. V. Wang, M. Suter, and J. F. Meinel, "Development of the first bioluminescence CT scanner," Radiology, vol. 566, pp. 229, 2003.

[3] V. Ntziachristos, J. Ripoll, L. V Wang, and R. Weisslder, "Looking and listening to light: the evolution of whole body photonic imaging," Nat. Biotechnol., vol. 23, no. 3, pp. 313-320, March 2005.

[4] G. Wang, Y. Li, and M. Jiang, "Uniqueness theorems in bioluminescence tomography," Med. Phys., vol. 31, no. 8, pp. 2289-2299, August 2004.

[5] W. Cong, G. Wang, D. Kumar, Y. Liu, M. Jiang, L. V. Wang, E. A. Hoffman, G. McLennan, P. B. McCray, J. Zabner, and A. Cong, "Practical reconstruction method for bioluminescence tomography," Optics Express, vol. 13, no. 18, pp. 6756-6771, August 2005.

[6] Y. Lv, J. Tian, W. Cong, G. Wang, J. Luo, W. Yang, and $\mathrm{H}$. Li, "A multilevel adaptive finite element algorithm for bioluminescence tomography," Optics Express, vol. 14, pp. 8211-8223, 2006.

[7] A. J Chaudhari, F. Darvas, J. R Bading, R. A Moats, P. S Conti, D. J Smith, S. R Cherry, and R. M Leahy, "Hyperspectral and multispectral bioluminescence optical tomography for small animal imaging," Phys. Med. Biol., vol. 50, pp. 5421-5441, 2005.

[8] G. Alexandrakis, F. R Rannou, and A. F Chatziioannou, "Tomographic bioluminescence imaging by use of a combined optical-PET (OPET) system: a computer simulation feasibility study," Phys. Med. Biol., vol. 50, pp. 4225-4241, 2005.

[9] W. P. Segars, B. M. W. Tsui, E. C. Frey, G. A. Johnson, and S. S. Berr, "Development of a 4D digital mouse phantom for molecular imaging research," Mol. Imaging Biol., vol. 6, pp. 149-159, 2004.

[10] E. G. Birgin and J. M. Martinez, "Large-scale activeset box-constrained optimization method with spectral projected gradients," Comput. Optim. Appl., vol. 23, pp. 101-125, 2002.

[11] J. Bey, "Tetrahedral grid refinement," Computing, vol. 55, pp. 355-378, 1995.

[12] O. Coquoz, T. L. Troy, D. J.-McMullen, and B. W. Rice, "Determination of depth of in vivo bioluminescent signals using spectral imaging techniques," in Preceedings of SPIE Vol. 4967, pp. 37-45, 2003.

[13] Ziji Wu, Accurate and Efficient Three-Dimensional Mesh Generation for Biomedical Engineering Applications, Ph.D. thesis, Worcester Polytechnic Institute, 2001. 